\title{
Perceived Stigma Against Alzheimer's Disease in the Turkish Population
}

\author{
(1) Büşra Sümeyye Arıca Polat1, (1) Musa Temel2, (1) Nuriye Kayalı1, (1) Nedime Tuğçe Bilbay3 \\ 1 University of Health Sciences Turkey, Gülhane Training and Research Hospital, Clinic of Neurology, Ankara, Turkey \\ 2Şanlıurfa Training and Research Hospital, Clinic of Neurology, Şanlıurfa, Turkey \\ 3University of Health Sciences Turkey, Dışkapı Yıldırım Beyazıt Training and Research Hospital, Clinic of Neurology, Ankara, Turkey
}

\begin{abstract}
Objective: Cognitive, behavioral, and psychiatric disorders that are associated with dementia cause stigma against Alzheimer's disease (AD) in patients and caregivers as well as in healthy individuals in public. To the best of our knowledge, stigma against AD is not investigated in the Turkish population; therefore, this study aimed to evaluate the stigma of healthy Turkish people according to their demographic characteristics.
\end{abstract}

Materials and Methods: This cross-sectional observational study included a total of 439 healthy participants without any history of neurological and/or systemic disease. Demographic characteristics and AD-related knowledge of participants were recorded. A 10-item questionnaire survey was applied to the participants to assess the stigmatization against AD. Factors associated with the stigma score were evaluated in regression analysis.

Results: This study included 253 males and 186 females (mean age: $35.7 \pm 9.8$ years). Most of them had knowledge about AD (94.3\%). Only 18.5\% had a family history of $A D$, and 65\% were married. Of 439 persons, $95(21.6 \%)$ worked in healthcare fields. Of the healthy participants, 60.6\% had a moderate-high stigma against AD. The mean stigma score was $8.95 \pm 4.79$. The total stigma scores were higher in females and singles $(p=0.001$ and $p<0.001$, respectively). Healthcare workers expressed the highest levels of stigma $(p<0.001)$. Age, knowledge, and family history of $A D$ did not influence the stigma. Shame, loss of self-esteem, and fear of exclusion were most expressed stigmas.

Conclusion: To the best of our knowledge, this is the first study that evaluated perceived stigma against AD in the healthy Turkish population. The higher incidence of stigma among females and singles can be explained by cultural reasons. Stigma in health professionals may lead to delay in the early diagnosis and management of AD.

Keywords: Alzheimer's disease, stigma, healthcare workers, healthy population

\section{Introduction}

Alzheimer's disease $(A D)$ is a progressive neurodegenerative disease that presents with episodic memory impairment and gradually decreases other cognitive functions (1). The global prevalence of the disease, which increases twice every 5.5 years, is expected to be 114.5 million in $2050(2,3)$. Early recognition of this disease, which has a tremendous socio-economic impact worldwide, is important for increasing appropriate treatment of dementia and managing its psychological, emotional, familial, economic terms. However, the perceived stigma against AD is seen as a huge factor in preventing effective strategies for the disease (4).

Stigma is defined as the negative attitude of the public towards people who are thought to be different in terms of physical, mental, and lifestyle. People who carry this label are less desired (5). Patients with memory impairment are known to judge themselves due to their illness and symptoms and experience both social and internalized stigmatization (6). In addition, family members and caregivers have stigmatization also (7). Moreover, some people believe that AD patients are less fortunate than those with treatable diseases (8). Understanding

Address for Correspondence: Musa Temel, Şanlıurfa Training and Research Hospital, Clinic of Neurology, Şanlıurfa, Turkey Phone: +90 5333340722 E-mail: mdmusatemel@hotmail.com ORCID: orcid.org/0000-0002-4687-7276

Received: 21.10.2021 Accepted: 15.11.2021

Cite this article as: Arıca Polat BS, Temel M, Kayalı N, Bilbay NT. Perceived Stigma Against Alzheimer's Disease in the Turkish Population. Eur J Geriatr Gerontol 2022;4(1):32-36

- Copyright 2022 by the Academic Geriatrics Society / European Journal of Geriatrics and Gerontology published by Galenos Publishing House. 
the perception of stigma in healthy individuals and educating society with accessible and effective methods helps to increase disease-related awareness and improve disease management (9).

To the best of our knowledge, there is no study evaluating the perceived stigma against $A D$ in healthy people in Turkey. Therefore, this study aims to investigate the perceived stigma level against $A D$ in healthy people. Its second aim is to reveal the demographic characteristics and related factors of people with high stigmatization.

\section{Materials and Methods}

\section{Study population}

Four hundred thirty-nine healthy participants (253 men, 186 women) living in Turkey's capital Ankara were included in this cross-sectional observational study. Participants in the study had no known cognitive and/or systemic disorders. Participants' age, gender, marital status, educational status, and occupation were recorded. Participants were asked about knowledge and family history of AD.

\section{Survey}

A ten-question survey (Table 1) was applied to the participants to assess the perceived stigma against AD. This questionnaire is the adaptation of the "STIG-MA" survey developed by Piver et al. (10) in 2012, with the author's permission. Participants were asked to pretend that they had $A D$ and rate what they would felt. The questions were answered as "yes, maybe, I don't know, no" and scored between 0-3. The score 3 corresponded to the greatest stigma, either "yes" if the question was positive (questions 1, 3, 4, 5, 6, 7, and 9) or "no" if the question was negative (questions 2, 8 and, 10). Other answers were scored respectively. The total score indicated the stigma severity. The highest score was 30. Zero-seven was graded as mild, 8-11 as moderate, and 12 and above the high stigma.

The questions were also grouped to explore several dimensions of perceived stigma: Reluctance to disclose the illness (questions

Table 1 . Ten questions about perceived stigma in the stigma survey

If you were suffering from Alzheimer's disease

1. Would you rather people did not know about your disease?

2. Would you tell the person you are closest to?

3. Would you lose self-esteem because of the disease?

4. Would this disease cause you shame or embarrassment?

5. Would your neighbors, your colleagues have less respect for you?

6. Do you think others would avoid you because of the disease?

7. Would your neighbors, your colleagues have less esteem for your family?

8. Do you think your wife/husband would stay with you and support you?

9. Do you think people you know at work or friends would ask you to stay away, even if you were taking medication for the disease?

10 . Would your family give you their support right from the start?
1 and 2), emotional impact (questions 3 and 4), fear of exclusion (questions 5, 6, and 9), courtesy stigma (question 7) and fear of loss of family support (questions 8 and 10).

The study's ethics committee approval for the research and permissions for data sharing for scientific purposes was obtained (23.12.2020/96537014). The Helsinki Declaration 2008 principles were conducted the study.

\section{Statistics}

Descriptive statistics were expressed as mean, standard deviation, median, minimum, and maximum for continuous data; and as count and proportion for categorical data. Categorical data were analysed with the chi-square or Fisher's Exact tests. The distribution normality of the continuous variables was calculated with the Shapiro-Wilk test. We analysed the two groups with independent samples t-test for the normally distributed variables and with the Mann-Whitney $U$ test for the non-normally distributed variables. We compared the more than two groups with One-Way ANOVA for the normally distributed variables and with the Kruskal-Wallis test for the non-normally distributed variables; post-hoc multiple comparison analysis was performed with significant values that have been adjusted by the Bonferroni correction. Pearson's or Spearman's correlation analysis was used to find the relationship between continuous variables, depending on the distribution.

Statistical analyses were performed using the IBM SPSS v.24 for Windows software and was reported with 95\% confidence intervals. Values of $p<0.05$ were considered significant.

\section{Results}

Four hundred thirty-nine healthy participants (186 females (42.4\%), 253 males (57.6\%), mean age 35.7 years \pm 9.8 ) without neurological and/or systemic diseases were included in this study. $94.3 \%(n=414)$ of the participants stated that they have knowledge about AD. But only 18.5\% $(n=81)$ had a family history of AD. $95(21.6 \%)$ of the participants were healthcare workers. Also, 152 (34.6\%) participants were singles.

According to the STIG-MA survey, the mean stigma score of the participants against $A D$ was $8.95 \pm 4.79$. $60.6 \%$ of healthy participants have a moderate-high stigma against $A D$. Mean scores of the dimensions of perceived stigma are summarized in Table 2.

Participants were divided into three groups according to their scores from this questionnaire: mild stigma $(n=173,39.4 \%)$, moderate stigma $(n=133,30.3 \%)$, and severe stigma $(n=133$, $30.3 \%$ ). $31.8 \%$ of the participants in the mild stigma group, $50.4 \%$ of those in the moderate stigma group, and $48.1 \%$ of those in the severe stigma group were women. Moderate and severe stigma scores were significantly higher in women than in men $(p=0.001)$. The mean stigma score was higher in singles 


\begin{tabular}{l}
\begin{tabular}{|l|l|}
\hline $\begin{array}{l}\text { Table 2. Global stigma score and five dimensions of perceived } \\
\text { stigma }\end{array}$ \\
\hline Global stigma score $(\mathrm{M} \pm \mathrm{SD})$ & $8.95( \pm 4.79)$ \\
\hline Reluctance to disclose the illness $(\mathrm{M} \pm \mathrm{SD})$ & $0.93( \pm 1.44)$ \\
\hline Emotional impact $(\mathrm{M} \pm \mathrm{SD})$ & $3.00( \pm 2.18)$ \\
Fear of exclusion $(\mathrm{M} \pm \mathrm{SD})$ & $3.67( \pm 2.49)$ \\
Courtesy stigma $(\mathrm{M} \pm \mathrm{SD})$ & $0.72( \pm 1.01)$ \\
Fear of loss of family support $(\mathrm{M} \pm \mathrm{SD})$ & $0.60( \pm 1.01)$ \\
\hline M: Mean, SD: Standart deviation &
\end{tabular} \\
\hline
\end{tabular}

$(p<0.001)$. Moderate-severe stigma was detected in $72(75.8 \%)$ of 95 healthcare workers participating in the study, and the stigma in healthcare workers was found to be statistically significant compared to non-healthcare workers $(p<0.001)$. Healthcare workers had higher stigma scores than non-healthcare workers. The three groups were similar in terms of age, knowledge about $A D$, family history of $A D$, and occupation (Table 3 ).

Correlation analysis was applied to investigate the relationship between dimensions of perceived stigma against AD and age, gender, AD awareness, marital status and occupation. The stigma scores of healthcare workers in all dimensions were higher than those without, and this result was statistically significant (respectively; $p=0.006, p=0.034, p<0.001, p=0.021, p=0.009$ ). It was observed that women got higher scores in the dimensions of "reluctance to declare the illness", "emotional impact" and "loss of family support" ( $p=0.018, p<0.001, p<0.001$, respectively). In singles, stigma scores were higher in the dimensions of "emotional impact" and "loss of family support" ( $p=0.006$, $p<0.001)$. Other factors did not have a significant effect on the dimensions of the perceived stigma.

\section{Discussion}

Our study has shown that $60.6 \%$ of healthy participants have a moderate-high stigma against AD. Stigma levels were higher in women, singles, and healthcare workers.

Older people with cognitive impairment encounter stigmatization in many cultures. Stigmatization causes a delay of 1.5-1.8 years in the diagnosis of cognitive impairment (11). It has been reported that older people are stigmatized due to their age, and additional cognitive impairment increases the risk of stigmatization threefold (12). Cognitive, behavioral, and psychiatric disorders associated with dementia cause some issues such as denial, embarrassment, and irritability associated with $A D$ in both patients, caregivers, and healthy individuals in the community. In Western societies, dementia is seen as a loss of youth, functionality, and independence. This, in turn, is associated with reduced tolerance and increased risk of stigma for dementia patients (13). In Eastern countries such as Japan, China, and India, dementia is perceived not as a disease but as a part of aging. Therefore, unlike in Western societies, the fear of having $A D$ and the frequency of stigma associated with it are less common in these countries (14-16). While some AfricanAmericans think of dementia as the inevitable consequence of aging, others define it as a mental illness because of their spiritual and religious beliefs. Therefore, these populations experience less stigma, caregiver burden, and caring problems against $A D(17,18)$. In Arabic countries, because of prohibitions on disclosing personal and family issues to foreigners, Alzheimer's patients' access to hospitals has decreased, resulting in criticism and ridicule (19).

On the other hand, solid religious beliefs increase compassion and the willingness to help patients with $A D$ and reduce aggression towards patients (13). In a study conducted with a population of $A D$ and caregivers, approximately two-thirds of the participants stated that they were not understood by others and were exposed to negative associations in society (20). In our study, the perceived stigma against $A D$ was high in more than half of the healthy Turkish participants. While this rate is similar to some studies reported in Western societies, it is higher than the stigma in Eastern cultures. This difference may be due to the cultural factors and demographic characteristics of the participants.

Some factors affect people's perception of stigma, such as gender, age, education level, cultural beliefs, knowledge about $A D$, or being an $A D$ caregiver. The most important factor associated with the perceived stigma against $A D$ is gender (21). In a study from Australia, stigmatization against $A D$ was found to be higher in men. However, women have three times more risk in elderly societies with a higher male population and gender

Table 3. Stigma levels of the participants according to their demographic characteristics

\begin{tabular}{|c|c|c|c|c|}
\hline & $\begin{array}{l}\text { Mild stigma } \\
(n=173)\end{array}$ & $\begin{array}{l}\text { Moderate stigma } \\
(n=133)\end{array}$ & $\begin{array}{l}\text { High stigma } \\
(n=133)\end{array}$ & p \\
\hline $\mathrm{Age}^{* *}$ (year), $(\mathrm{M} \pm \mathrm{SD})$ & $36.8( \pm 10.3)$ & $35.3( \pm 10.3)$ & $34.7( \pm 8.6)$ & 0.242 \\
\hline Gender ${ }^{* * *}$, female $n,(\%)$ & $55(31.8)$ & $67(50.4)$ & $64(48.1)$ & $0.001^{*}$ \\
\hline $\begin{array}{l}\text { Knowledge about } A D^{* * *}, n_{1}(\%) \\
\text { Family history with } A D^{* * *}, n_{1}(\%) \\
\text { Marital status }{ }^{* *} \text {, single } n,(\%) \\
\text { Occupation }{ }^{* * *}, \text { worker } n,(\%) \\
\text { Heathcare worker }{ }^{* * *}, n_{1}(\%)\end{array}$ & $\begin{array}{l}14(8.1) \\
35(20.2) \\
40(23.1) \\
156(90.2) \\
23(13.3)\end{array}$ & $\begin{array}{l}5(3.8) \\
27(20.3) \\
52(39.1) \\
115(86.5) \\
28(21.1)\end{array}$ & $\begin{array}{l}6(4.5) \\
19(14.3) \\
60(45.1) \\
126(94.7) \\
44(33.1)\end{array}$ & $\begin{array}{l}0.062 \\
0.903 \\
\mathbf{0 . 0 0 0 *} \\
0.071 \\
\mathbf{0 . 0 0 0 *}\end{array}$ \\
\hline
\end{tabular}


discrimination $(22,23)$. In our study, perceived stigma against AD was found to be higher in women than in men. This result was thought to be related to the fact that women are more interested in the care of AD patients in our country, and therefore women are more exposed to the behavioral effects of the disease.

Studies have shown that the perception of stigma in young people is higher than in the elderly $(22,24)$. Different from previous studies, although most of our participants were young, age did not have any effect on stigmatization in our study.

The socio-economic factors and education level affect stigmatization (25). In high educated and income countries, cognitive impairments are known as an unavoidable part of normal aging. This increases fear and anxiety in individuals, feelings of hopelessness, and stigmatization (26). In the low income countries, people accept patients with AD more easily and stigmatize them less (25). In some studies, it has been shown that education level does not affect stigma $(21,27)$. Similarly, no relationship was found between marital status and stigmatization (28). In our study, the stigma level was not affected by the education level and the occupation. However, in contrast to the literature, it was observed that single participants got higher scores in the "Fear of loss of family support" and "Emotional impact" dimensions. The high stigmatization in these dimensions is thought to be due to people not having any idea about their future partners and their behavior.

Although the vast majority of the participants (94.3\%) included in our study have knowledge about $A D$, they had a high stigma against the disease. This result is different from other studies reporting the relationship between having less knowledge about the disease and an increased risk of stigma. People who have more information about AD have less stigma (29). In a study conducted in 155 countries with more than 70.000 participants, it was found that two-thirds of the participants accepted $A D$ as a part of the aging process, not as a neurodegenerative disease. This indicates the lack of knowledge about $A D$ that may result in stigma (30).

There was no difference in stigma between those who had a family member with dementia and those who did not $(21,24,31)$. Only about one-fifth of our participants had a family history of $A D$, which was not associated with stigma, consistent with previous studies.

It has been demonstrated that approximately $62 \%$ of healthcare workers know dementia as a part of normal aging in a large study (32). Education of healthcare professionals about AD and increasing their awareness about the disease is necessary for early diagnosis of $A D$ and developing effective strategies to manage it. Thus, national dementia outcomes could be improved (33). A few studies have shown that the perception of stigma against $A D$ is high among healthcare professionals $(10,32)$. Our study also revealed a high stigma against $A D$ in most healthcare workers.
To the best of our knowledge, our study is the first to investigate the perceived stigma prevalence against $A D$ in the healthy Turkish population. However, the study has some limitations. Participants are generally young people, so stigmatization in older individuals is not clear. Besides, since AD caregivers were not included in this study, their stigmatization could not be evaluated.

\section{Conclusion}

Perceived stigma against $A D$ is frequent in healthy Turkish individuals and is higher in women, singles, and healthcare workers. The prevalence of stigma may vary between different cultures. Stigma not only delays the diagnosis of $A D$, but also prevents patients from accessing medical and surgical interventions, making decisions about their own care and legal processes, and also expressing opinions on $A D$ treatment and support. It is important to provide national strategies for eliminating the perception of stigma. Similar evaluations have been made before, especially in high- and low-income societies. Still, such an evaluation has never been made before in a middle income country such as Turkey. Therefore, our study is valuable because it shows the levels of stigma against $A D$ in Turkish society, especially according to occupational groups, and such an evaluation has not been made before. Nevertheless, there is a need for large-scale studies investigating the frequency of stigma and related factors in the general population.

\section{Information}

This study was previously published on the preprint (Authorea) server. Doi:10.22541/au.161973544.40611837/v1

\section{Ethics}

Ethics Committee Approval: The study's ethics committee approval for the research and permissions for data sharing for scientific purposes was obtained (23.12.2020/96537014). The Helsinki Declaration 2008 principles were conducted the study.

Informed Consent: All volunteers participating in the survey were informed about the study and agreed to participate. Since there was no patient group, an extra voluntary consent form was not obtained.

Peer-review: Externally peer-reviewed

\section{Authorship Contributions}

Surgical and Medical Practices: M.T., Concept: N.K., B.S.A.P., Design: N.K., B.S.A.P., Data Collection or Processing: M.T., N.T.B., B.S.A.P., Analysis or Interpretation: M.T., N.K., N.T.B., B.S.A.P., Literature Search: M.T., N.T.B., B.S.A.P., Writing: M.T., B.S.A.P.

Conflict of Interest: No conflict of interest was declared by the authors. 
Financial Disclosure: The authors declared that this study received no financial support.

\section{References}

1. McKhann GM, Knopman DS, Chertkow H, Hyman BT, Jack CR Jr, Kawas CH, Klunk WE, Koroshetz WJ, Manly JJ, Mayeux R, Mohs RC, Morris JC, Rossor MN, Scheltens P, Carrillo MC, Thies B, Weintraub S, Phelps $\mathrm{CH}$. The diagnosis of dementia due to Alzheimer's disease: recommendations from the National Institute on Aging-Alzheimer's Association workgroups on diagnostic guidelines for Alzheimer's disease. Alzheimers Dement 2011;7:263-269.

2. Fiest KM, Roberts Jl, Maxwell CJ, Hogan DB, Smith EE, Frolkis A, Cohen A, Kirk A, Pearson D, Pringsheim T, Venegas-Torres A, Jetté N. The Prevalence and Incidence of Dementia Due to Alzheimer's Disease: a Systematic Review and Meta-Analysis. Can J Neurol Sci 2016;43(Suppl 1):S51-82.

3. Prince $M$, Bryce $R$, Albanese $E$, Wimo A, Ribeiro $W$, Ferri CP. The global prevalence of dementia: a systematic review and metaanalysis. Alzheimers Dement 2013;9:63-75.e2.

4. Lobo A, Launer $\sqcup$, Fratiglioni L, Andersen K, Di Carlo A, Breteler MM, Copeland JR, Dartigues JF, Jagger C, Martinez-Lage J, Soininen H, Hofman A. Prevalence of dementia and major subtypes in Europe: A collaborative study of population-based cohorts. Neurologic Diseases in the Elderly Research Group. Neurology 2000;54(11 Suppl 5):S4-9.

5. Jolley DJ, Benbow SM. Stigma and Alzheimer's disease: causes, consequences and a constructive approach. Int J Clin Pract 2000;54:117-119.

6. Stites SD, Milne R, Karlawish J. Advances in Alzheimer's imaging are changing the experience of Alzheimer's disease. Alzheimers Dement (Amst) 2018;10:285-300

7. Cavayas $M$, Raffard $S$, Gély-Nargeot MC. Stigmatisation dans la maladie d'Alzheimer, une revue de la question [Stigmatization in Alzheimer's disease, a review]. Geriatr Psychol Neuropsychiatr Vieil 2012;10:297-305.

8. Alzheimer's Disease International (ADI) \& In The Moment (ITM) Productions. (2018). [The Global Voice on Dementia]. Every three seconds. [Video file]. Retrieved from https://www.alz.co.uk/everythreeseconds.

9. The World Health Organization (WHO). (2017). 10 facts on dementia. From https://www.who.int/features/factfiles/dementia/en/

10. Piver LC, Nubukpo P, Faure A, Dumoitier N, Couratier P, Clément JP. Describing perceived stigma against Alzheimer's disease in a general population in France: the STIG-MA survey. Int J Geriatr Psychiatry 2013;28:933-938.

11. de Miranda LFJR, Matoso RO, Rodrigues MV, de Lima TOL, Nascimento AF, Carvalho FC, Moreira DRM, Fernandes JC, de Paula JJ, Magno LAV, Caramelli $\mathrm{P}$, de Moraes EN. Factors influencing possible delay in the diagnosis of Alzheimer's disease: Findings from a tertiary Public University Hospital. Dement Neuropsychol 2011;5:328-331.

12. Benbow SM, Reynolds D. Challenging the stigma of Alzheimer's disease. Hosp Med 2000;61:174-177.

13. Cipriani G, Borin G. Understanding dementia in the sociocultural context: a review. Int J Soc Psychiatry 2015;61:198-204.

14. Traphagan JW. Senility as disintegrated person in Japan. J Cross Cult Gerontol 2002;17:253-267.

15. Sun $F$, Ong $R$, Burnette $D$. The influence of ethnicity and culture on dementia caregiving: a review of empirical studies on Chinese Americans. Am J Alzheimers Dis Other Demen 2012;27:13-22.

16. Dias A, Dewey ME, D'Souza J, Dhume R, Motghare DD, Shaji KS, Menon R, Prince $M$, Patel V. The effectiveness of a home care program for supporting caregivers of persons with dementia in developing countries: a randomised controlled trial from Goa, India. PLoS One 2008;3:e2333.
17. Mahoney DF, Cloutterbuck J, Neary S, Zhan L. African American, Chinese, and Latino family caregivers' impressions of the onset and diagnosis of dementia: cross-cultural similarities and differences. Gerontologist 2005; $45: 783-792$.

18. Roberts JS, Connell CM, Cisewski D, Hipps YG, Demissie S, Green RC. Differences between African Americans and whites in their perceptions of Alzheimer disease. Alzheimer Dis Assoc Disord 2003;17:19-26.

19. Youssef J, Deane FP. Factors influencing mentalhealth help-seeking in Arabic-speaking communities in Sydney, Australia. Mental Health Religion ct Culture 2006;9:43-66.

20. Batch ML, Mittelman, MS. World Alzheimer Report 2012: Executive Summary. 2012.

21. Rewerska-Juśko M, Rejdak K. Social Stigma of People with Dementia. J Alzheimers Dis 2020;78:1339-1343.

22. Kim S, Mortby M. Dementia-related stigma, cultural diversity and helpseeking intentions: Findings from a population survey. Innov Aging 2017;1(Suppl 1):693.

23. Graham N, Lindesay J, Katona C, Bertolote JM, Camus V, Copeland JR, de Mendonça Lima CA, Gaillard M, Gély Nargeot MC, Gray J, Jacobsson L, Kingma M, Kühne N, O'Loughlin A, Rutz W, Saraceno B, Taintor Z, Wancata J; Old Age Psychiatry section, World Psychiatric Association; World Health Organization. Reducing stigma and discrimination against older people with mental disorders: a technical consensus statement. Int J Geriatr Psychiatry 2003;18:670-678.

24. Mazurek M, Papuć E. [Stigmatisation of people with dementi]. Wiad Lek 2018;71:1275-1278.

25. Pelegrini LNC, Hall A, Hooper E, Oliveira D, Guerra F, Casemiro FG, Bonfadini J, Yong K, Pereira N, Costa R, Barbosa MT, Mioshi E. Challenges in public perception: highlights from the United Kingdom-Brazil Dementia Workshop. Dement Neuropsychol 2020;14:209-215.

26. Rosenberg A, Coley N, Soulier A, Kulmala J, Soininen H, Andrieu S, Kivipelto $M$, Barbera $M$; MIND-AD and HATICE groups. Experiences of dementia and attitude towards prevention: a qualitative study among older adults participating in a prevention trial. BMC Geriatr 2020;20:99.

27. Werner $P$, Davidson M. Emotional reactions of lay persons to someone with Alzheimer's disease. Int J Geriatr Psychiatry 2004;19:391-397.

28. Liang J, Jang Y, Aranda MP. Stigmatising beliefs about Alzheimer's disease: Findings from the Asian American Quality of Life Survey. Health Soc Care Community 2021;29:1483-1490.

29. Stites SD, Largent EA, Johnson R, Harkins K, Karlawish J. Effects of SelfIdentification as a Caregiver on Expectations of Public Stigma of Alzheimer's Disease. J Alzheimers Dis Rep 2021;5:31-39.

30. Perel-Levin S. Stigma, dementia and age. World Alzheimer Report 2019: Attitudes to dementia 3.2, 2019;90-92.

31. Woo BK. Family history and its relationship with dementia stigma beliefs among Chinese Americans. Geriatr Gerontol Int 2017;17:122-125.

32. World Alzheimer Report 2019, Attitudes to dementia https://www. alzheimer-waw.pl/wp-content/uploads/2019/09/World-Alzheimer-Report -2019-

33. Ibanez A, Flichtentrei $D$, Hesse $E$, Dottori M, Tomio A, Slachevsky A, Serrano CM, Gonzalez-Billaut C, Custodio N, Miranda C, Bustin J, Cetckovitch M, Torrente F, Olavarria L, Leon T, Beber BC, Bruki S, Suemoto CK, Nitrini R, Miller BL, Yokoyama JS. The power of knowledge about dementia in Latin America across health professionals working on aging. Alzheimers Dement (Amst) 2020;12:e12117. 